\title{
DESIGN COMPLEXITY AS A DRIVER FOR ADDITIVE MANUFACTURING PROCESS IMPROVEMENT
}

\author{
Nishkal George ${ }^{1^{*}}$ and Boppana V. Chowdary ${ }^{2}$ \\ ${ }^{1,2}$ Faculty of Engineering, The University of the West Indies, Trinidad \\ ${ }^{1}$ Email: nishkalgeorge@gmail.com*(Corresponding author) \\ ${ }^{2}$ Email: Boppana.Chowdary@ sta.uwi.edu
}

\begin{abstract}
Design complexity in additive manufacturing (AM) is a current issue in the research community, fueled by the well-known phrase "complexity for free". This statement has promoted the assumption that complex geometries may be achieved without any increase in the cost of production. However, recent research has indicated that increasing shape complexity produces an increase in production costs for the material extrusion process. This challenges the mainstream assumption that AM technologies provide 'complexity for free'. The AM community requires further investigation of design complexity and its impact on sustainable production when used as a Design for Manufacturing (DfM) tool. This paper proposes a data-driven method which uses design complexity as an AM performance indicator for the material extrusion process. The manufacturing responses included build time (BT), dimensional accuracy (DA) and complexity index (CI). Design space exploration of an automotive air filter model was achieved by varying five critical design features which impact complexity. The study utilized a Face Centered Central Composite Design (FCCCD) of three levels for the design features, comprising 32 experimental models. The optimal model was manufactured based on multi-objective optimization using the MINITAB $\odot$ response optimizer. This method exploits the design features to achieve target performance and manufacturability. The viability of design complexity as an AM performance indicator was discussed leading to three major improvements to the Product Design and Development (PDD) process for AM. The proposed improvements have the potential to reduce process times and minimize resources, providing a sustainable AM approach for developing regions.
\end{abstract}

Keywords: Additive Manufacturing, Design Complexity, Multi-objective optimization, Product Design and Development, Design for Manufacturing

https://doi.org/10.47412/HEXU4041

\section{Introduction}

AM technology has been purported as a revolutionary achievement for design and manufacturing. Despite the numerous advantages of AM, researchers are recently acknowledging that the AM process has unique challenges. The three major challenges facing AM users are shape optimization, design for 3D printing and pre and post-processing [1]. Poor utilization of the available design space creates issues for the manufacturing process including material and energy wastage which do not contribute to sustainable AM. Another factor which lends to the perception by end-users of the technology that these limitations are well controlled, is the use of the catchphrase "complexity for free" expressed in a largely popular and influential 
book where the authors proposed that complex geometries could be exploited in AM without additional increase in time and cost [2]. The lack of sustainable design strategies paired with the minimization of design as an issue puts additional pressure on machine setup, such as the reduction of layer thickness or increase in deposition speed, to correct the inadequacies of the design.

Thus, design strategies which improve the AM process have a growing interest to manufacturers with strict resource limits. The issue of incorporating intricate geometries in AM design has been an ongoing discussion, a significant theoretical explanation was proposed by [3]. The author indicates that perimeter travel distance paired with design volume is a significant factor of excessive manufacturing time in AM. This theoretical discussion was transformed into experimental work following these arguments, which attempted to determine the implications of complex geometry in AM. One study provided a generous assessment of the effects of complexity with respect to 3D sand printing which had yet to be achieved for the material extrusion process using an optimization approach [4]. Following this, an AM case study focusing on the material extrusion process proposed a method to quantify the complexity of material extrusion processed parts [5]. The method utilized design data based on a Shape Complexity Index, a ratio of part surface area to the bounding box volume. The study conducted an experiment on a load cell holder with five variations of shape complexity and examined the impact on part cost.

Thus far, the experimental work has only achieved to conclude that design complexity in AM is a cost component that requires more detailed investigation. This study proposes that this gap can be filled by a thorough statistical design space exploration of a component and furthermore describes its implications to the AM PDD process. Design space exploration has been described as a data-driven design method defined as the search of potential solutions which meet design targets within a specified range [6]. The study concluded that a multidisciplinary data-driven optimization method was able to effectively design simultaneously for product performance, material and manufacturing process. Data-driven methods provide rapid search of potentially optimal designs through design exploration and exploitation [6]. Therefore, through the combination of a data-driven design method such as multi-objective optimization paired with the previously employed complexity index, an optimal design solution for target manufacturability may be attained.

\section{Method}

The methodology explores the statistical relationships between design features and the responses of design complexity, build time and dimensional accuracy. The design data is gathered through design space exploration using experimental models. The optimal design which achieved target performance and manufacturability was determined through design exploitation in the form of data-driven optimization. The initial geometry of the component being optimized is a simplified version of an automotive air filter frame, shown in Fig. 1. The indicated design features are described in Table 1.

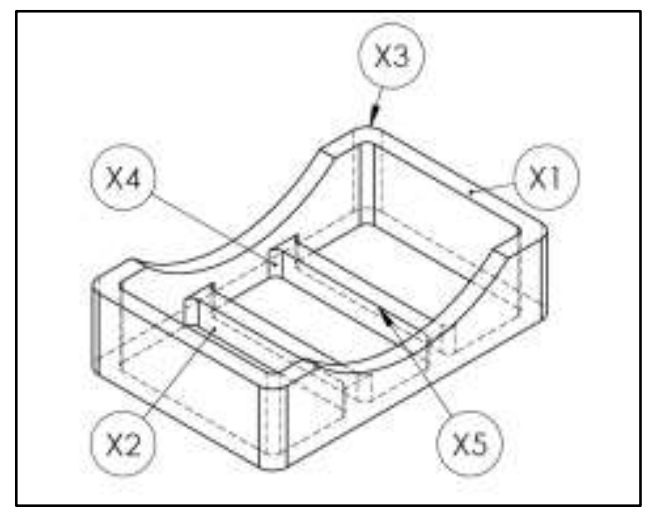


Figure 1: Design features of air filter model.

The shape consists of two functional surfaces at either side which contact with the filter housing, with the available design space between the functional surfaces. Recent studies have utilized this concept of functional surfaces as limits for the enclosed design volume (DV) [5,7]. This study identified five design features in accordance with the design attributes of the part. These design features include; wall thickness (WT), internal fillet (IF), external fillet (EF), number of ribs (NR) and rib height (RH). For each experiment, the Surface Area (SA) and Design Volume (DV) were used to calculate the Complexity Index (CI) as shown in Eq. (1). The complexity index served as the primary objective function.

$$
C I=\frac{S A}{D V}
$$

Dimensional accuracy (DA), as proposed in this study, utilized the method of direct observation of a single axis using a statistical form demonstrated by for the relative deviation in dimension [8], as shown in Eq. (2).

$$
\Delta Z=\left|\frac{Z_{A C T U A L}-Z_{C A D}}{Z_{C A D}}\right|
$$

$\mathrm{Z}_{\mathrm{ACTUAL}}$ represents measured value of dimension, $\mathrm{Z}_{\mathrm{CAD}}$ represents the respective $\mathrm{CAD}$ model value, $\Delta \mathrm{Z}$ represents relative deviation in Z. Built time (BT) was measured from the actual time from the initial extrusion to the completion of the model. A Face Centred Central Composite Design (FCCCD) was used to generate the experimental models. FCCCD is a fractional factorial $2^{\mathrm{K}}$ design, which comprises of $\mathrm{K}$ factors at coded levels of $+1,-1$ and 0 for the uncoded maximum, minimum and centre values of each factor respectively as shown in Table 1 .

Table 1: Design feature levels for FCCCD.

\begin{tabular}{|c|c|c|c|c|c|}
\hline $\begin{array}{c}\text { Factor } \\
\text { (Design Feature) }\end{array}$ & Symbol & Unit & $\begin{array}{c}\text { Low Level } \\
(-1)\end{array}$ & $\begin{array}{c}\text { Centre } \\
\text { point }(0)\end{array}$ & $\begin{array}{c}\text { High Level } \\
(+1)\end{array}$ \\
\hline Wall thickness (WT) & $\mathrm{X} 1$ & $\mathrm{~mm}$ & 1.5 & 3.25 & 5 \\
\hline Number of Ribs (NR) & $\mathrm{X} 2$ & $\mathrm{count}$ & 2 & 3 & 4 \\
\hline External fillet (EF) & $\mathrm{X} 3$ & $\mathrm{~mm}$ & 0 & 1.5 & 3 \\
\hline Internal fillet (IF) & $\mathrm{X} 4$ & $\mathrm{~mm}$ & 0 & 1.5 & 3 \\
\hline Rib height (RH) & $\mathrm{X} 5$ & $\mathrm{~mm}$ & 3 & 5 & 7 \\
\hline
\end{tabular}

For this study, a Raise $3 \mathrm{D} ® \mathrm{~N} 2$ Plus printer using $1.75 \mathrm{~mm}$ filament was utilized, the build envelope for this printer is 12"x12"x24". A total of 32 experiments were modeled in the Solidworks® 2016 package, sliced using the ideaMaker ${ }^{\circledR}$ software and printed using the material extrusion process parameters shown in Table 2.

Table 1: Material extrusion process parameters.

\begin{tabular}{|c|c|c|}
\hline No. & Process Parameter & Value \\
\hline 1 & Bed Temperature & $110^{\circ} \mathrm{C}$ \\
\hline 2 & Extruder Nozzle & $225^{\circ} \mathrm{C}$ \\
\hline 3 & Infill Percentage & $10 \%$ \\
\hline 4 & Layer Height & $0.15 \mathrm{~mm}$ \\
\hline 5 & Infill Speed & $60 \mathrm{~mm} / \mathrm{s}$ \\
\hline 6 & Shells & 2 \\
\hline 7 & First Layer Speed & $15 \mathrm{~mm} / \mathrm{s}$ \\
\hline 8 & First Layer Height & $0.3 \mathrm{~mm}$ \\
\hline
\end{tabular}




\begin{tabular}{|c|c|c|}
\hline 9 & Infill Pattern & Grid \\
\hline 10 & Brim & 8 lines \\
\hline
\end{tabular}

\section{Results}

\subsection{Design Exploration}

For this study, design exploration is achieved through ANOVA of the raw design data. This analysis was used to produce regression models and main effects plots from which inferences about the optimal design features for the air filter could be derived. The regression models obtained for CI, BT and DA shown Eq. 3 to Eq. 5 were deemed satisfactory for further statistical analysis and optimization. The regression models include design feature interactions through linear, quadratic and second-order polynomial terms.
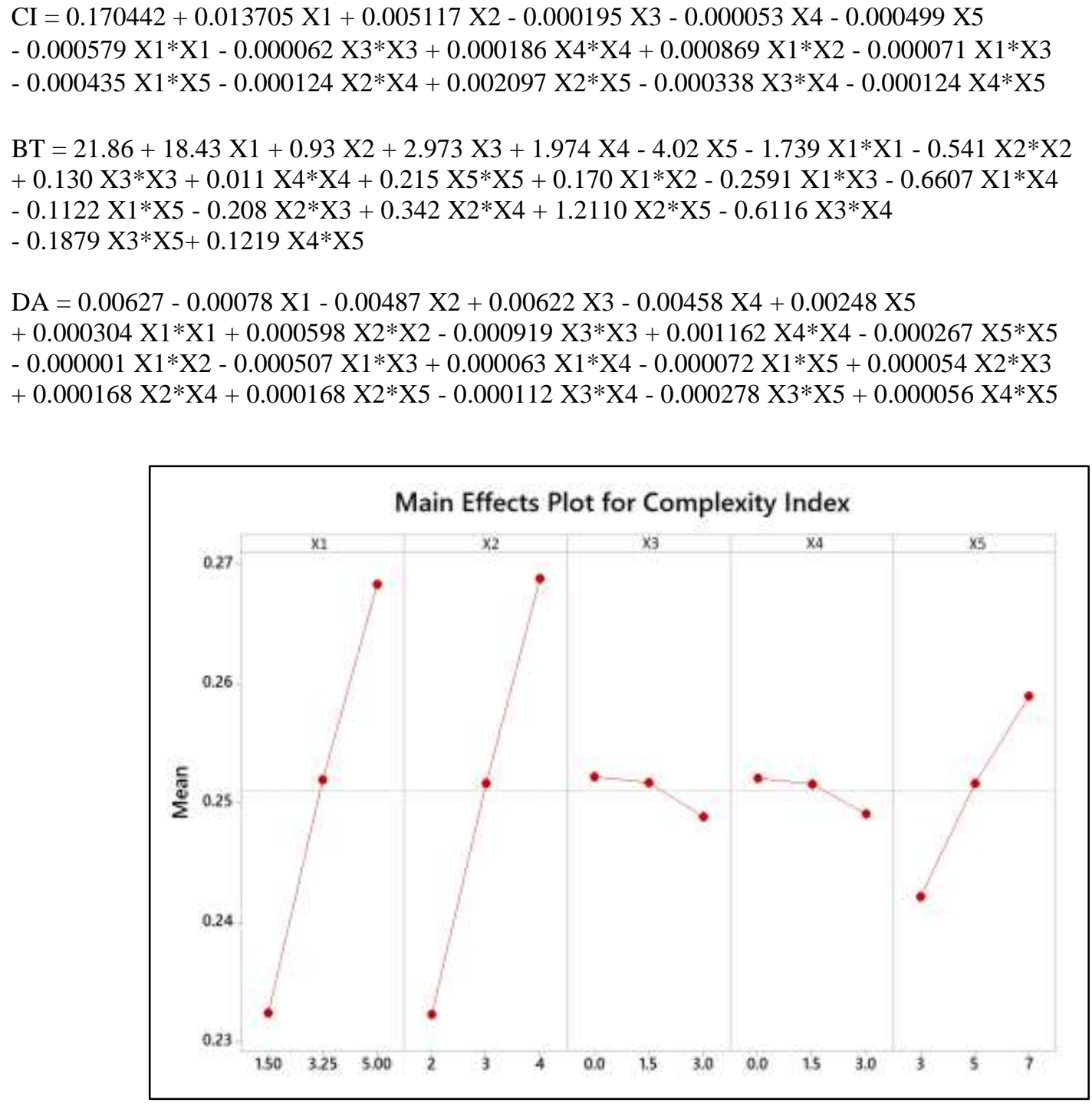

Figure 2: Main effects plot for CI.

The Main effects plot for CI shown in Fig. 2, provided a preliminary assessment on the influence of the independent design features on CI. It was observed that X1 and X2 were the largest contributors to increase 
in CI, with a linear increase being observed for both factors. Design feature X5 showed similar increase while $\mathrm{X} 3$ and $\mathrm{X} 4$ produced a marginal decreasing effect when varied from minimum to maximum. The Main effects plot for EBT shown in Fig. 3 suggests that X1 and X2 are the largest contributors to increase in EBT. Design feature X1 was found to produce the largest increase followed by X2 while X3, X4 and X5 indicate that unwanted conditions exist in the central region of those design feature levels.

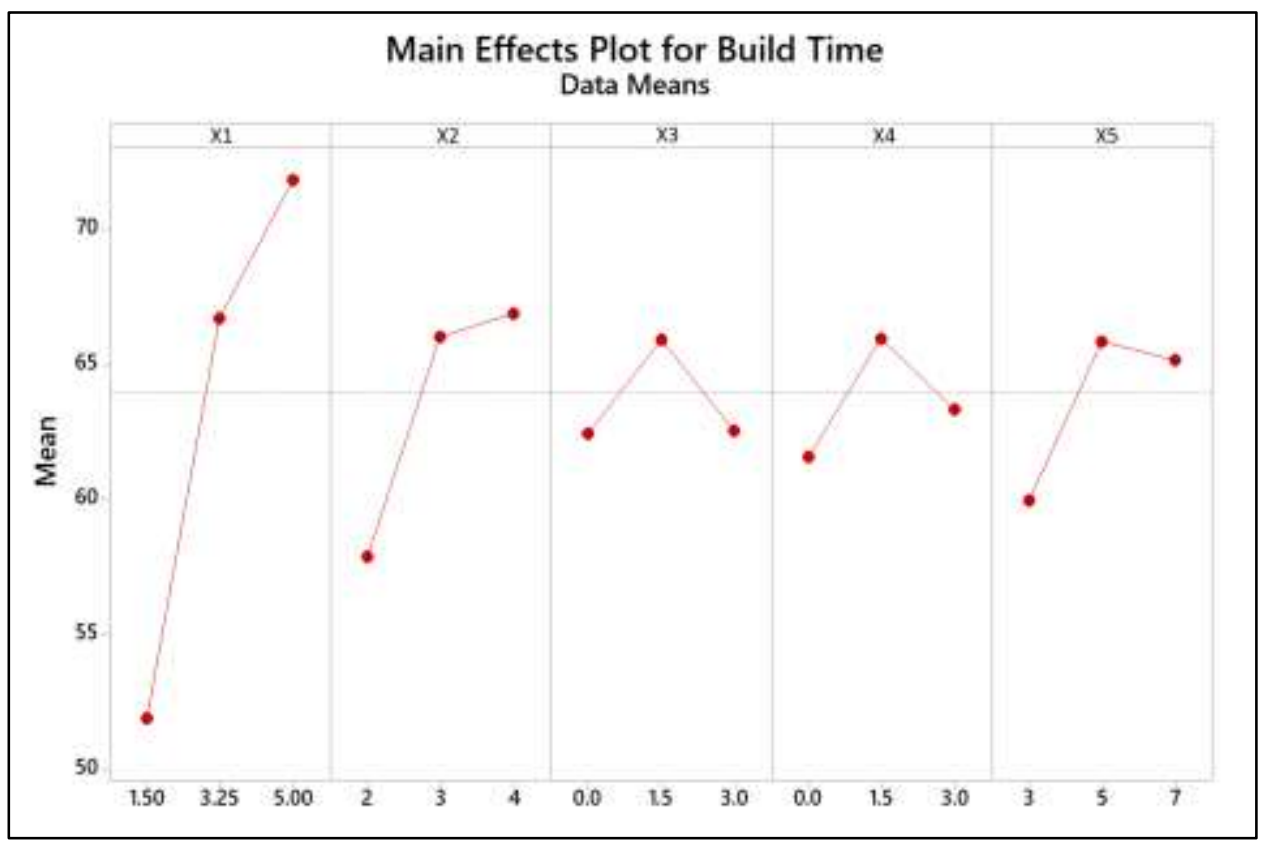

Figure 3: Main effects plot for BT.

The Main effects plot for DA is shown in Figure 4 suggests that all design features influence dimensional accuracy in the z-axis differently. Design features X3 and X4 produce the largest overall effect, with the latter producing the highest deviation of 0.0063 . However, $\mathrm{X} 1$ is the largest contributor to increase in deviation with an observed increase of 0.0013 for a single increase in X1 from $3.25 \mathrm{~mm}$ to $5 \mathrm{~mm}$. The preliminary statistical analysis reveals multiple options for response optimization. However, to encompass the interactions indicated in the regression models and improve sensitivity, response optimization is independent of the inferences made of the main effects. 


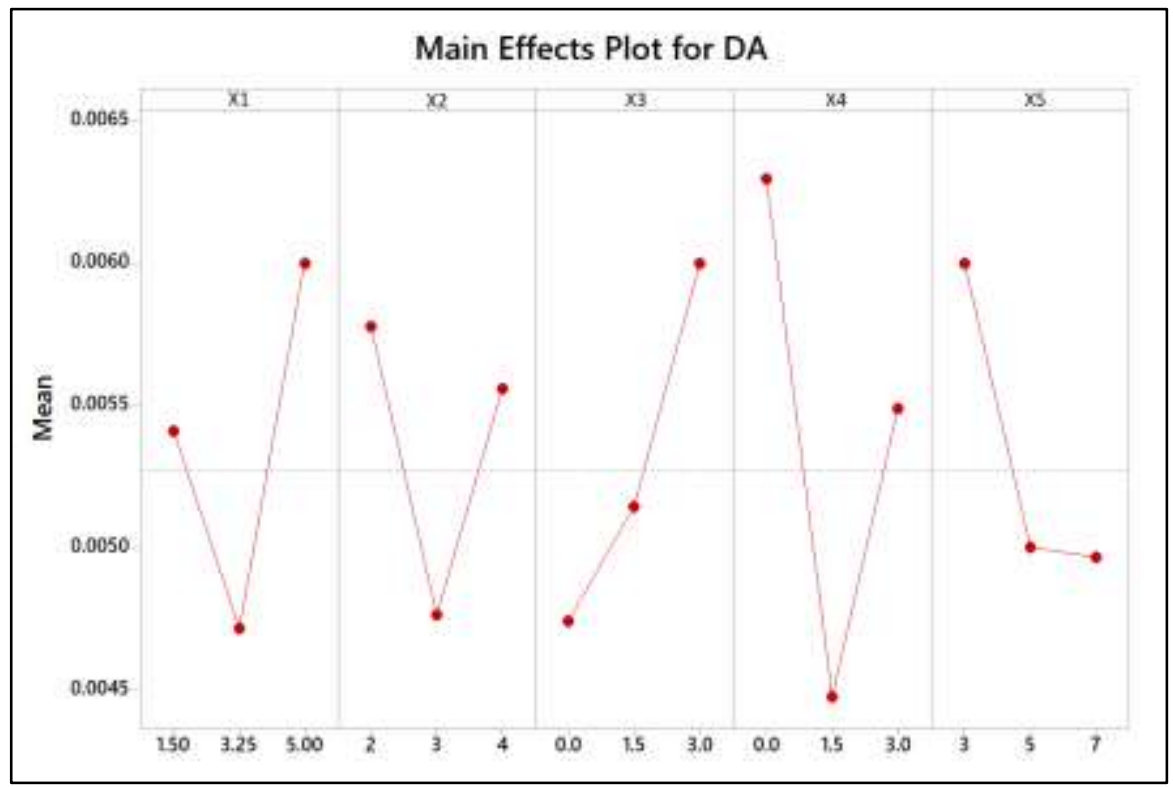

Figure 4: Main effects plot for DA.

\subsection{Design Exploitation and Optimization}

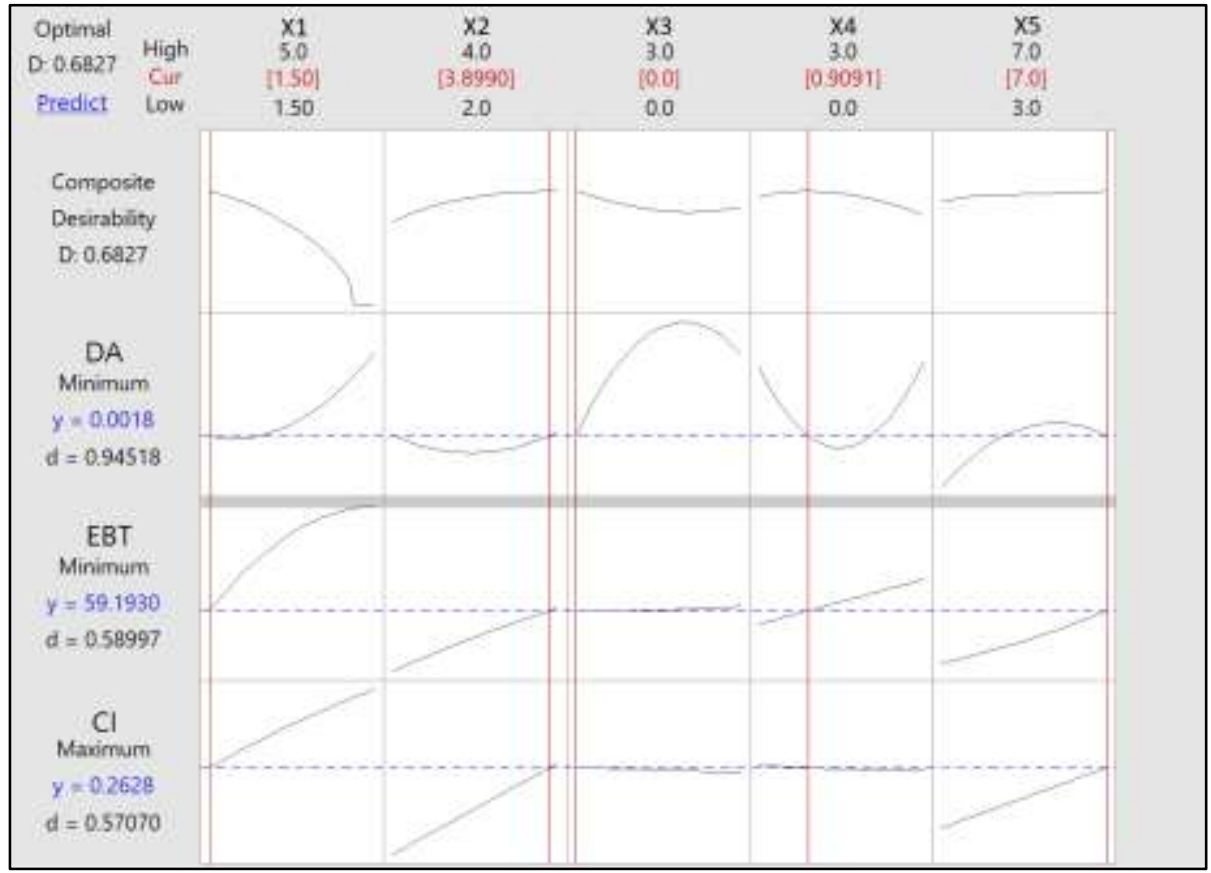

Figure 5: Response Optimizer results.

The MINITAB ${ }^{\circledR} 17$ Response Optimizer results, shown in Fig. 5, further validated the observed effects during the design exploration. The optimized design feature levels are necessary to exploit the design to reach target design values. In this study, the highest manufacturability is required, represented by setting the response optimizer parameters to obtain maximum CI, minimal BT and minimal DA. The composite desirability plots show that minimum (-1) levels of X1 and X3 were the most desirable for all responses, while the maximum (+1) levels for X2 and X5 were the most desirable. The optimization results shown in the plot indicate that the preferred design feature configuration is $1.5 \mathrm{~mm}, 0 \mathrm{~mm}, 0.9 \mathrm{~mm}$ and $7 \mathrm{~mm}$ for $\mathrm{X} 1$, 
$\mathrm{X} 3, \mathrm{X} 4$ and $\mathrm{X} 5$ respectively with an X2 of approximately 4. The highest complexity for the best manufacturing performance for the air filter model was reached at a CI of 0.263 , which corresponds to a BT of 59.19 minutes and a DA of 0.002 . When compared to a non-optimal model of the same model mass with no additional support material, the MINTAB® response optimized model showed a $10 \%$ reduction in BT from 66.73 for a non-optimized model with equal mass of $6.1 \mathrm{~g}$.

\section{Discussion and Findings}

\subsection{Design Optimization}

The study proposed to develop a data-driven method which adequately determines the optimal CI or complexity limit for the case of an automotive air filter model. Design exploration of the individual design features of the air filter model was achieved through statistical analysis and main effects plots. The combination of individual design features was exploited to produce maximum manufacturability for maximum complexity. This method can facilitate the creation and management of realistic design target values for performance and manufacturability simultaneously by balancing multiple criteria.

As three manufacturing criteria were investigated in this study, a multi-objective optimization technique was used. The MINITAB 17 response optimizer was capable of producing a model which is fabricated 10\% faster with increased accuracy as compared to non-optimal design of identical mass. The reduction is marginal for a single air filter model, however, for continuous production it can significantly increase production output and cost savings. The findings suggest that manufacturing requirements can be effectively controlled for AM using CI.

\subsection{AM Process Improvement}

Design management was proposed as the realization of the design process through organizational and management activities [9], with the goal of producing low cost products with high manufacturability and quality. The results suggest that the proposed data-driven method utilizing design complexity can enhance the traditional PDD process shown in Fig. 6. This is achieved by bridging the gap between design features of the $\mathrm{CAD}$ phase and $\mathrm{AM}$ performance responses. With the addition of mathematical models of the design features to the CAD phase, feedback loops of the responses for AM performance using EBT and Quality Control using DA were made. The designer may exploit specific design feature configurations utilizing CI to improve the AM responses. This is summarized by three major enhancements made to the traditional PDD process for AM as shown in Fig. 7.

1) DA data was used to increase the accuracy of the model in the chosen axis through optimization. The feedback from QC allows the mathematical model for DA to be continuously improved by comparing theoretical and actual responses.

2) The feedback of BT from AM production can allow design feature adjustments to be made to correct production issues such as production over time or idle time, one of the six categories of production loss that impact overall equipment effectiveness (OEE).

3) $\mathrm{AM}$ response optimization in the CAD phase is also fundamentally connected to AM process planning, as process planning typically involves identification of the production constraints. A maximum allowable BT, derived from the desired production volume, will influence the optimal values for CI and BT. The decreased reliance on RP parameter configuration, to obtain faster or slower production depending on the desired responses, also reduces machine setup and adjustment times. This can enhance OEE when producing in batches or allow for faster changeovers when using flexible manufacturing. 


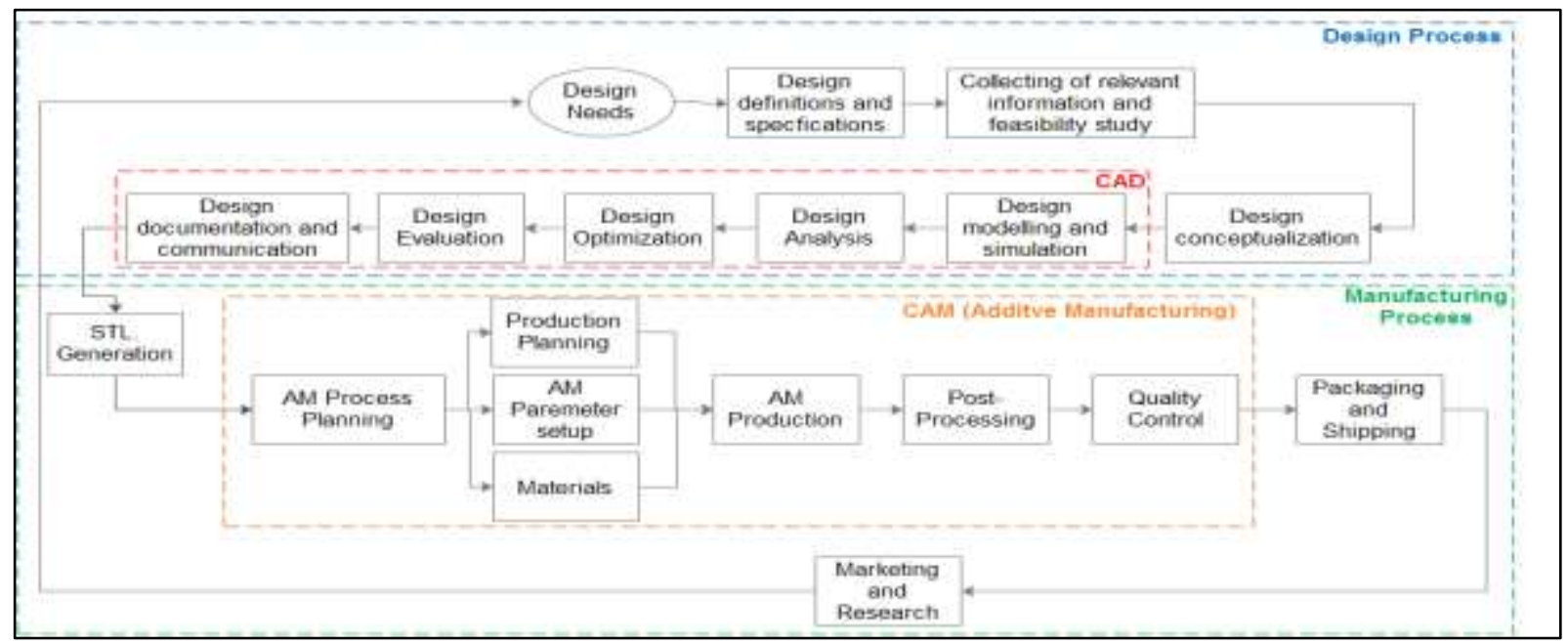

Figure 6: Typical educational PDD process for AM.

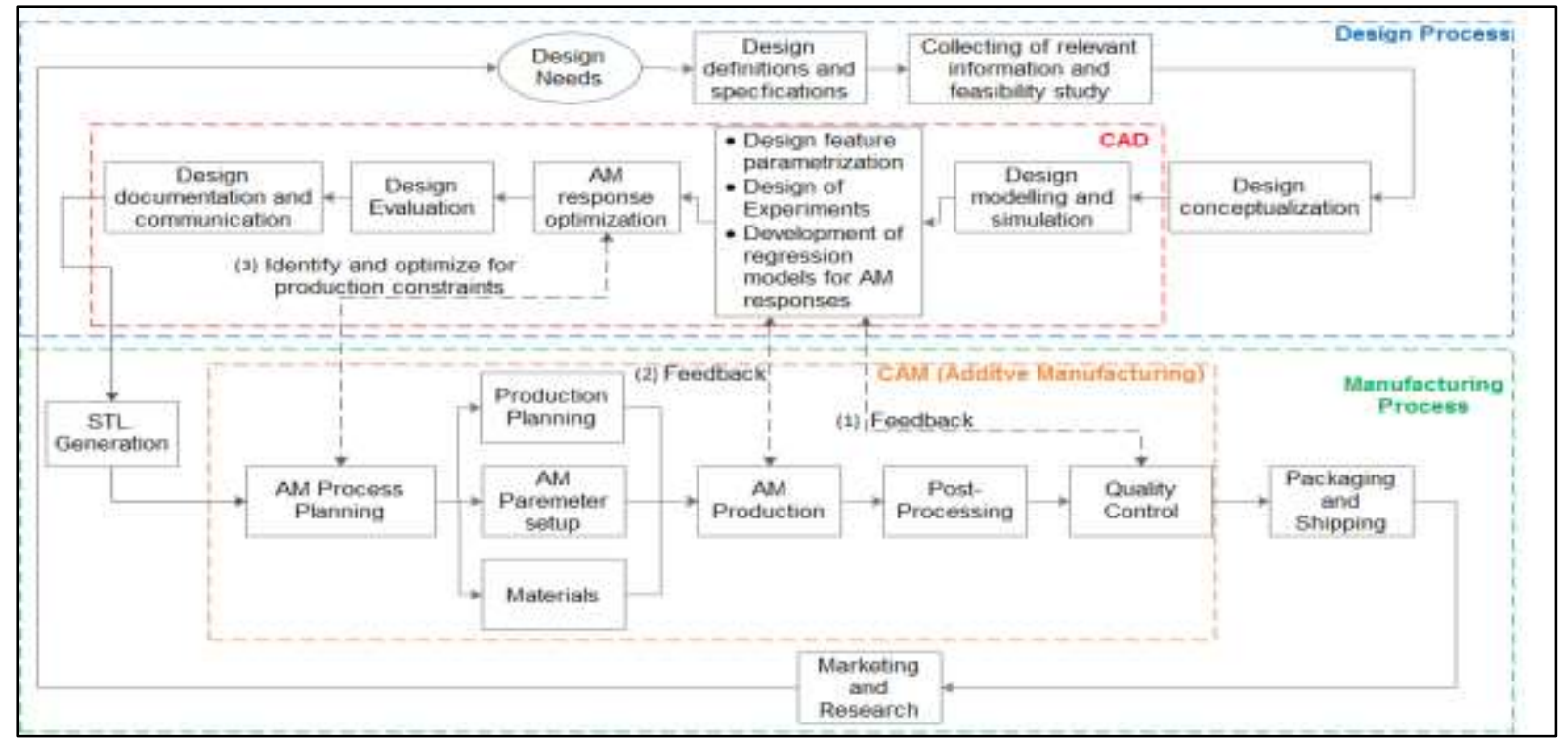

Figure 7: Design optimization enhanced PDD process for AM.

\section{Conclusion}

The initial challenge addressed in this study is design space exploration and exploitation using design complexity, while attempting to achieve target performance and manufacturability. Design complexity in AM was found to be a useful metric for assessing the manufacturing performance of a part. The findings suggest that design complexity, when monitored in conjunction with other manufacturing responses, may be used to enhance the PDD process for AM. Design exploration using a data-driven method incorporating design complexity was found to be an effective method for achieving this. The Complexity Index, CI was found to adequately represent the variation of complex design features of the automotive air filter model. The results indicate that all three investigated responses CI, BT and DA were optimized using the developed method. Further design exploitation through multi-objective optimization was performed by which BT for the air filter model was significantly reduced by $10 \%$ and DA increased for identical material usage. The 
proposed method successfully improves upon that proposed by [5] as it defines the variables of the AM component and fully quantifies the impact of each design feature and their interactions.

The identification of critical design features in this study, though based on literature, may be described methodically and subsequently improved. Thus, further work is needed to expand this methodology to other AM components and provide a framework for identifying and managing critical design features. As this study is fundamentally connected to a fixed design space volume, some maximum and minimum limits for design features can be obtained quantitatively. However, some design feature limits are based on designer discretion. Thus, distinguishing between these design features is another direction for future work.

\section{Acknowledgements}

The authors would like to thank for the Campus Research and Publication (CRP) Fund, for granting funds to this project (Ref. CRP.3.MAR18.2).

\section{References}

[1] Oropallo, William, and Les A. Piegl. 2015. "Ten challenges in 3D printing." Engineering with Computers 32 (1):135-148. doi: 10.1007/s00366-015-0407-0.

[2] Gibson, I, DW Rosen, and B Stucker. 2010. "Additive manufacturing technologies rapid prototyping to direct digital manufacturing. 2010." Springer.

[3] R. J. Urbanic and R. Hedrick, "Fused Deposition Modelling Design Rules for Building Large, Complex Components," Comput. Aided. Des. Appl., pp. 1-21, 2015.

[4] Almaghariz, Eyad S., Brett P. Connor, Lukas Lenner, Gullapalli. Ram, Guha P. Monogharan, Brandon Lamoncha, and Maureen Fang. 2016. "Quantifying the role of part design complexity in using 3D sand printing for molds and cores." International Journal of Metalcasting 10 (3):240-252. doi: 10.1007/s40962016-0027-5.

[5] Pradel, P., R. Bibb, Z. Zhu, and J. Moultrie. 2017. "Complexity Is Not for Free: The inpact of component complexity on additive manufacturing build time." Rapid Design, Prototyping and Manufacturing (RDPM) 2017, Newcastle.

[6] Xiong, Yi, Pham Luu Trung Duong, Dong Wang, Sang-In Park, Qi Ge, Nagarajan Raghavan, and David W Rosen. 2019. "Data-Driven Design Space Exploration and Exploitation for Design for Additive Manufacturing." Journal of Mechanical Design 141 (10):101101.

[7] Huang, Renkai, Ning Dai, Dawei Li, Xiaosheng Cheng, Hao Liu, and Dengguang Sun. 2018. "Parallel non-dominated sorting genetic algorithm-II for optimal part deposition orientation in additive manufacturing based on functional features." Proceedings of the Institution of Mechanical Engineers, Part C: Journal of Mechanical Engineering Science 232 (19):3384-3395.

[8] Sood, Anoop Kumar, RK Ohdar, and Siba Sankar Mahapatra. 2009. "Improving dimensional accuracy of fused deposition modelling processed part using grey Taguchi method." Materials \& Design 30 (10):4243-4252.

[9] Gorb, Peter, and Angela Dumas. 1987. "Silent design." Design studies 8 (3):150-156. 